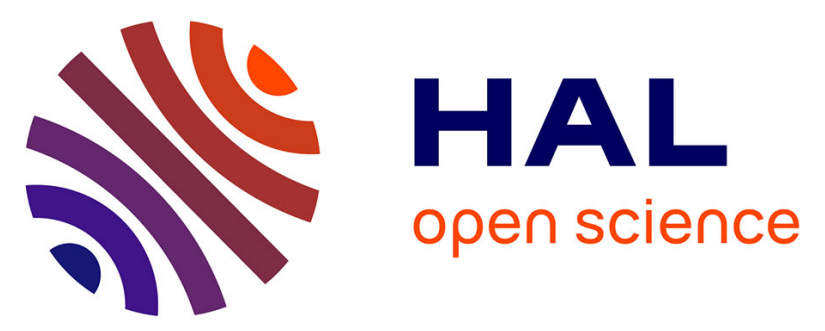

\title{
Asymmetric interhemispheric excitability evidenced by event-related potential amplitude patterns after "wide-awake surgery" of brain tumours
}

François Bonnetblanc, Guillaume Herbet, Pom Charras, Mitsuhiro Hayashibe, David Guiraud, Hugues Duffau, Bénédicte Poulin-Charronnat

\section{To cite this version:}

François Bonnetblanc, Guillaume Herbet, Pom Charras, Mitsuhiro Hayashibe, David Guiraud, et al.. Asymmetric interhemispheric excitability evidenced by event-related potential amplitude patterns after "wide-awake surgery" of brain tumours. Experimental Brain Research, 2014, 232 (12), pp.39073918. 10.1007/s00221-014-4075-x . lirmm-01103379

\section{HAL Id: lirmm-01103379 \\ https://hal-lirmm.ccsd.cnrs.fr/lirmm-01103379}

Submitted on 14 Jan 2015

HAL is a multi-disciplinary open access archive for the deposit and dissemination of scientific research documents, whether they are published or not. The documents may come from teaching and research institutions in France or abroad, or from public or private research centers.
L'archive ouverte pluridisciplinaire HAL, est destinée au dépôt et à la diffusion de documents scientifiques de niveau recherche, publiés ou non, émanant des établissements d'enseignement et de recherche français ou étrangers, des laboratoires publics ou privés. 


\title{
Asymmetric interhemispheric excitability evidenced by event-related potential amplitude patterns after "wide-awake surgery" of brain tumours
}

\author{
François Bonnetblanc • Guillaume Herbet • \\ Pom Charras • Mitsuhiro Hayashibe · David Guiraud • \\ Hugues Duffau · Bénédicte Poulin-Charronnat
}

Received: 27 March 2014 / Accepted: 11 August 2014

(C) Springer-Verlag Berlin Heidelberg 2014

\begin{abstract}
Slow-growing, infiltrative brain tumours may modify the electrophysiological balance between the two hemispheres. To determine whether and how asymmetry in interhemispheric excitability might occur following "wide-awake surgery" for this type of tumour, we recorded electroencephalograms during a simple visuo-manual reaction time paradigm performed by five patients between 3 and 12 months after surgery. Interhemispheric excitability asymmetries were computed by comparing the amplitudes of event-related potentials (ERPs) in the injured hemisphere to those in the healthy hemisphere. For the two patients with the smallest lesions $\left(7.1\right.$ and $11.5 \mathrm{~cm}^{3}$, respectively), increased excitability within the ipsilesional hemisphere was evidenced by characteristics increases in
\end{abstract}

F. Bonnetblanc $\cdot$ P. Charras $\cdot$ M. Hayashibe $\cdot$ D. Guiraud INRIA, LIRMM, équipe DEMAR, University of Montpellier 2, 34095 Montpellier, France

\section{F. Bonnetblanc $(\square)$}

Cognition, Action et Plasticité Sensorimotrice, INSERM U-1093, UFR STAPS, Université de Bourgogne, 27877 Dijon, France e-mail: francois.bonnetblanc@u-bourgogne.fr

\section{F. Bonnetblanc}

Institut Universitaire de France, 75005 Paris, France

G. Herbet $\cdot$ H. Duffau

Départment de Neurochirurgie, Hôpital Gui de Chauliac,

34295 Montpellier, France

G. Herbet $\cdot$ H. Duffau

Institut des Neurosciences de Montpellier, INSERM U-1051,

Hôpital Saint Eloi, 34091 Montpellier, France

B. Poulin-Charronnat $(\square)$

LEAD - CNRS UMR5022, Université de Bourgogne,

27877 Dijon, France

e-mail: benedicte.poulin@u-bourgogne.fr the ERP amplitude at several sites, with few occurrences in the contralesional hemisphere. For smaller lesions (and under certain experimental conditions), cortical excitability in the injured hemisphere may increase in order to maintain local compensation. In addition, we observed and increased excitability in the contralesional frontal homologue for one patient who underwent an extensive resection. Postoperative monitoring of interhemispheric asymmetries in ERP amplitudes is of value for determining task constraints inducing electrophysiological imbalance and guiding rehabilitation.

Keywords Wide-awake surgery $\cdot$ Slow-growing infiltrative brain tumours $\cdot$ Plasticity $\cdot$ Interhemispheric imbalance $\cdot$ Cortical excitability

\section{Introduction}

Real-time functional mapping of the brain with direct electrical stimulation (DES) is used to guide the resection of slow-growing infiltrative tumours during wide-awake surgery. The DES technique reduces the probability of resecting essential areas near or within the tumour (Duffau 2005). During wide-awake surgery, patients perform a number of neuropsychological tests while DES is applied both cortically and sub-cortically, in order to detect and thus preserve connectivity online (especially for critical white matter pathways). This technique enables functional mapping of the area near the tumour so that as much as non-eloquent infiltrated tissue as possible can be removed while minimizing sequelae.

Slow-growing lesions prompt much greater functional brain reorganization than acute lesions do. More precisely, the recruitment of remote brain areas in the ipsi- and 
contralesional hemispheres may be more efficient. As such, the assessment of recovery in slow-growing versus acute lesions may offer new interesting insights into brain plasticity (Finger 1978; Finger and Stein 1982; Stein et al. 1983; Bonnetblanc et al. 2006; Desmurget et al. 2007). The brain's impressive reorganizational capacity enables patients with slow-growing tumours to recover substantially within days, hours (Bonnetblanc et al. 2006; Desmurget et al. 2007; Herbet et al. 2013) or even minutes of surgery (Sallard et al. 2012a). These impressive, rapid compensations may be partially explained by the progressive emergence of compensatory networks in the contralesional hemisphere as those of the ipsilesional hemisphere are disrupted by slow-growing tumour. Hence, surgical removal of the tumour may rapidly unmask homologous contralesional networks.

Although direct observations [using functional magnetic resonance imaging (fMRI) or transcranial magnetic stimulation (TMS)] have suggested that the contralesional hemisphere is involved in functional recovery, the topic needs further investigation. In particular, Krainik et al. (2004) used fMRI to show the involvement of a contralesional homologue after resection of the supplementary motor area. However, the significance of these functional translocations is subject to debate. Indeed, recruitment of the intact contralesional homologue may reflect a decrease in transcallosal inhibition (Netz et al. 1995; Heiss et al. 2003). In contrast, a more recent study has rebutted these concerns by showing that TMS induces speech disruption when applied to contralesional activation sites (Thiel et al. 2005). Taken as a whole, these results do not mean that the ipsilesional hemisphere has a minor or inexistent role in the recovery process. In fact, one hypothesis concerning functional compensation during slow tumour growth suggests that compensatory networks could switch progressively from the ipsilesional hemisphere to the contralesional hemisphere as the lesion increases in size (Bonnetblanc et al. 2006; Desmurget et al. 2007). For focal acute lesions (induced experimentally in animals or observed in stroke patients), early increases in cortical excitability in the ipsilesional hemisphere have been observed in the vicinity of the lesion (and more specifically in the motor cortex), which is suggestive of acute functional reorganization in this area (Jacobs and Donoghue 1991; Nudo et al. 1996; Sanes and Donoghue 2000; Hummel and Cohen 2005, 2006). However, to the best of our knowledge, changes in excitability have not been monitored in patients harbouring slow-growing tumours, and interhemispheric imbalance has not been evaluated after the resection of large volumes from a single hemisphere. On the behavioural level, some preliminary research on the responses to a visual "go" signal three or 4 days after surgery has evidenced longer reaction times for the impaired hand than for the healthy hand (Sallard et al. 2012b). These hand function asymmetries are suggestive of some degree of interhemispheric imbalance.

To further document (1) the role of each hemisphere in this compensation and (2) possible electrophysiological imbalances after surgery, we measured the electroencephalographic (EEG) activity of patients performing an analogue visuo-manual reaction time paradigm between 3 and 12 months after surgery. We hypothesized that the postlesional interhemispheric imbalance could be evidenced by characteristic changes in cortical excitability (as measured by EEG) in general and interhemispheric imbalance in event-related potential (ERP) amplitudes in particular. Importantly, we measured cortical excitability by recording ERPs (rather the classical motor or sensory evoked potentials) in an ecological visuo-manual reaction time paradigm. In theory, each hemisphere's involvement in the latter task varies as a function of the location of the stimulus and the hand used to react. For instance, the role of the left hemisphere in task performance will supposedly be greatest for a stimulus displayed in the right hemifield and for a response given with the right hand. The use of other combinations of the visual stimulus location and the responding hand will supposedly enable the experimenter to access each hemisphere to a different extent. Consequently, we also sought to determine whether the measured changes in interhemispheric excitability were correlated (at least to some extent) with estimations provided by various stimulus location/hand-used combinations.

The brain reorganization that occurs during slow lesion growth and then after resection of a large part of the brain is critical in many aspects. On the electrophysiological level, dynamic aspects of the brain may be considerably altered, and EEG signals are necessarily modified by the presence of the cavity. The EEG analyses that are performed usually on healthy subjects or patients with a complete brain volume (as well as pre- vs. post-operative comparisons in resected patients) can only be performed if the signal distortion (due to the cavity) is removed by complex modelling of the lesion and signal conduction pathways. To circumvent these problems and concentrate on excitability changes in the injured and healthy hemispheres, we focused on post-operative comparisons. This approach to understanding interhemispheric dynamics is fully valid, since it is also performed in stroke patients (from whom no pre-lesion data are available). To exclude complex changes in the dynamics of ERP signals (due to signal distortion and changes in sources location), we simply computed an asymmetry index by comparing the ERP amplitudes at pairs of homologous electrodes. Hence, simple post-operative analyses of ERP amplitudes enabled us to investigate asymmetries in interhemispheric excitability. 


\section{Materials and methods}

Participants

Five right-handed patients (P1 to P5; 4 males and 1 female; mean \pm SD age $39 \pm 5.34$, range $=[33 ; 45])$ and eight agematched, right-handed healthy controls ( 7 males and 1 female; mean age $39.75 \pm 4.30$, range $=[33 ; 45])$ participated in the study. None of the controls had a history of neurological disease (including motor, visual hemifield or visuospatial impairments). The study complied with the World Medical Association's Code of Ethics (the 1964 Declaration of Helsinki and its amendments). The patients' socio-demographic and clinical characteristics are summarized in Table 1.

P1 (a 38-year-old male) presented with a right temporal tumour (Fig. 1a). P2 and P3 (a 44-year-old female and a 33-year-old male, respectively) presented with a right frontal tumour (Fig. 1b, c). P4 (a 35-year-old male) presented with a tumour at right middle and inferior temporal area. P5 (a 45-year-old male, respectively) presented with a tumour in the right temporal pole (Fig. 1d, e). All patients underwent the surgical resection of low-grade glioma (grade II, according to the World Health Organization classification) with intra-operative DES.

Lesion mapping was based on whole-brain three-dimensional T1 anatomical MRI data sets acquired 3 months after surgery (on a 3T Siemens Skyra machine, Siemens Medical Systems, Erlangen, Germany). These individual MRI data sets were then normalized against the Montreal Neurological Institute template by applying cost function masking (Brett et al. 2001). This method has been very well characterized and is known to be the best option for normalization

Table 1 Socio-demographic and clinical data

\begin{tabular}{|c|c|c|c|c|c|c|c|c|}
\hline \multicolumn{5}{|c|}{ Socio-demographic } & \multicolumn{3}{|l|}{ Clinical } & \multirow{2}{*}{$\begin{array}{l}\text { Time of assessment after } \\
\text { the surgery (months) }\end{array}$} \\
\hline Id. & Age (years) & Gender & $\begin{array}{l}\text { Edu. level } \\
\text { (years) }\end{array}$ & Handed ness & $\begin{array}{l}\text { Grade of } \\
\text { the tumour }\end{array}$ & Surgery & $\begin{array}{l}\text { Volume of resection } \\
\left(\mathrm{cm}^{3}\right)\end{array}$ & \\
\hline P1 & 38 & M & 15 & $\mathrm{R}$ & II & Awake & 79.2 & 3 \\
\hline $\mathrm{P} 2$ & 44 & $\mathrm{~F}$ & 12 & $\mathrm{R}$ & II & Awake & 119.1 & 3 \\
\hline P3 & 33 & M & 17 & $\mathrm{R}$ & II & Awake & 116.8 & 9 \\
\hline P4 & 35 & M & 17 & $\mathrm{R}$ & II & Awake & 7.1 & 3 \\
\hline P5 & 45 & M & 17 & $\mathrm{R}$ & II & Awake & 11.5 & 12 \\
\hline
\end{tabular}

Fig. 1 Lesion mapping results for each patient (a) Patient 1

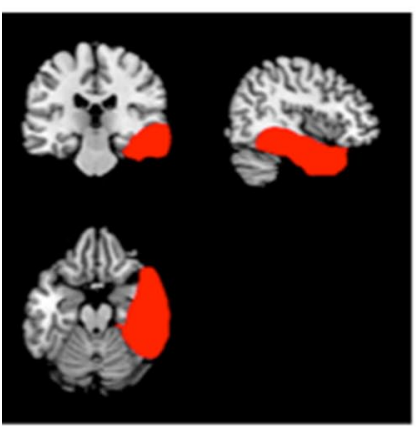

L $\mathbf{R}$

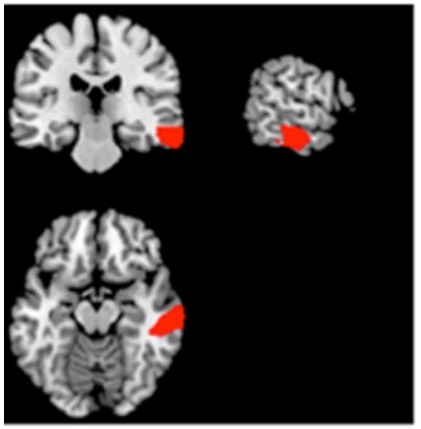

(d) Patient 4 (b) Patient 2

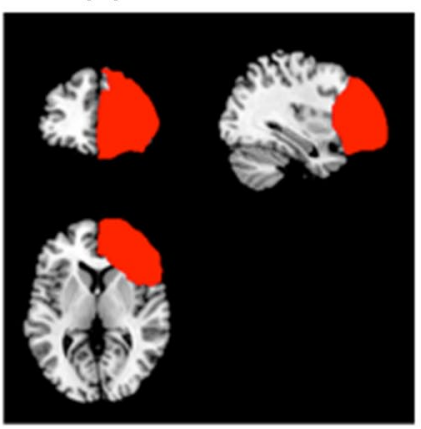

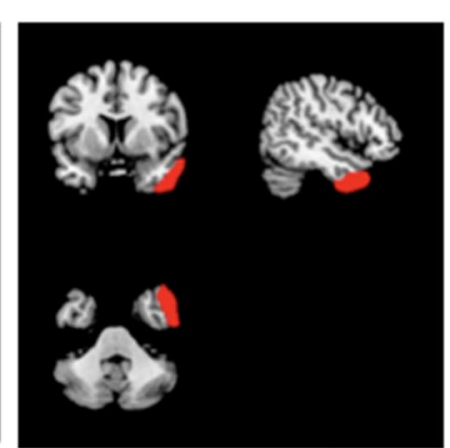

(e) Patient 5 (c) Patient 3

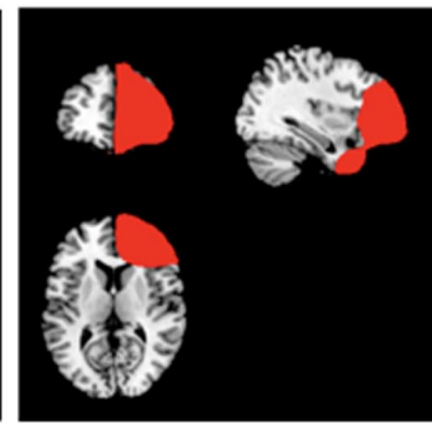


when the lesion is extensive (Andersen et al. 2010). During the registration process, cost function masking avoids the bias caused by abnormal, lesion-induced radiological signals. Briefly, the lesion was contoured (using MRIcron software (http://www.mccauslandcenter.sc.edu/mric ro/mricron) and transformed into a binarized image. This image was inserted during the normalization processes performed here with SPM 8 (http://www.fil.ion.ucl.ac.uk/spm/ software/spm8). The lesion is then redrawn on the normalized scan, in order to generate an individual volume of interest (illustrated in Fig. 1 for each patient).

\section{Clinical characteristics of the study population}

Patients were thoroughly examined by a neuropsychologist before and after surgery. None had clinically evident neurological disorders. Data on surgical resection volumes are summarized in Table 1. Three patients had extensive resections $\left(79.2,119.1\right.$ and $\left.116.8 \mathrm{~cm}^{3}\right)$, and two had much smaller resections $\left(7.1\right.$ and $\left.11.5 \mathrm{~cm}^{3}\right)$. P2 was undergoing her second surgical operation on the frontal lobe. The first operation had been performed in 2004 by another neurosurgeon, but the lesion had continued to grow in the intervening time.

\section{The behavioural task}

Participants comfortably sat in front of a laptop computer on which different visual stimuli (including the "go" signal) were displayed with Presentation ${ }^{\circledR}$ software (http://www.neurobs.com). The screen was about $60 \mathrm{~cm}$ from the body in the sagittal axis and $45 \mathrm{~cm}$ below eye level. Two switches were positioned on each side of the sagittal axis $(15 \mathrm{~cm}$ apart and $45 \mathrm{~cm}$ from the body). The motor responses consisted of pressing one or both switches with the corresponding index finger(s). A fixation cross appeared in the middle of the screen and served as the warning signal. After a variable time interval, one or two arrows appeared at different locations (represented the "go" signal); the participant sawing one or two arrows then had to press the corresponding button(s) as soon as possible. The arrow(s) could appear to the left of the initial fixation cross (designated as LS), to the right (RS), to both the left and the right at the same time (R + LS) or at the fixation cross's central position (centre, CS). The time interval between the appearance of the fixation cross and the appearance of the "go" signal (i.e. the stimulus onset asynchrony) varied randomly from one trial to another in the range 1,000 to 2,000 ms. This interval comprised two phases. Firstly, the fixation cross was displayed for a duration between 1,000 and $1,500 \mathrm{~ms}$. After the disappearance of fixation cross, the stimulus appeared after a variable interval of between 0 and $500 \mathrm{~ms}$. The four combinations of visual stimuli $(R S, L S, R+L S$ and $C S)$ were randomized within three blocks that depended on the hand(s) used (i.e. RH, $\mathrm{LH}$ and $\mathrm{R}+\mathrm{LH}$ ), giving a total of twelve conditions. A total of 360 trials ( 30 trials $\times 12$ conditions) were performed in two separate sessions.

\section{Event-related potential recording and pre-processing}

The electroencephalogram was recorded at 64 scalp sites using a 10-20 system (BioSemi ActiveTwo). Horizontal eye movements were recorded with electrodes placed on the outer left and right canthi. Vertical eye movements were recorded with an electrode placed under the right eye. Two additional electrodes were placed on the left and right mastoids (A1, A2). During recording, the BioSemi system's common mode sense electrode served as the reference electrode. Electrophysiological signals were digitized at a 2,048 $\mathrm{Hz}$ sampling rate and acquired with ActiView software. The EEG signals were analysed using the EEGLAB toolbox for Matlab. The data were re-referenced against the mean of electrodes A1 and A2, downsampled to $250 \mathrm{~Hz}$ and high-pass filtered with a cut-off frequency of $1 \mathrm{~Hz}$. In order to correct for eye movement artefacts, EEGLAB's binica routine was used to perform independent component analyses by removing the first component. All the corrections underwent visual inspection. For each condition, each patient's ERPs were averaged over a latency window going from $-1,000 \mathrm{~ms}$ prestimulus to $400 \mathrm{~ms}$ post-stimulus.

\section{Data analyses}

Behavioural and ERP data for each individual patient were compared with the $95 \%$ confidence interval (95\% CI) determined for the control group. In order to compare the ERP amplitudes in the right (injured) and left (healthy) hemispheres, we computed an asymmetry index (AI) for each pair of homologous electrodes and in each experimental condition: $\mathrm{AI}=-[|\mathrm{Right} \mathrm{ERP}|-\mid$ Left ERP on the homologous sitel]/the peak ERP at any site. To assess possible interhemispheric imbalances in cortical excitability in each experimental condition, we determined whether a given patient's AI was outside the $95 \%$ CI derived from the control group for a cumulative duration of $100 \mathrm{~ms}$ during a time window ranging from the stimulus onset $\left(\mathrm{t}_{0}=0 \mathrm{~ms}\right)$ to the motor response $(t \approx 400 \mathrm{~ms})$. As shown in Fig. 2 , a negative value (above the blue line, since the scales are inverted) meant that the normalized ERP amplitude in the right (injured) hemisphere (at $\mathrm{C} 4$, for instance) was higher than that at the homologous site (C3) and that the asymmetry exceeded that observed in healthy subjects. Conversely, a positive value (below the red line) meant that 


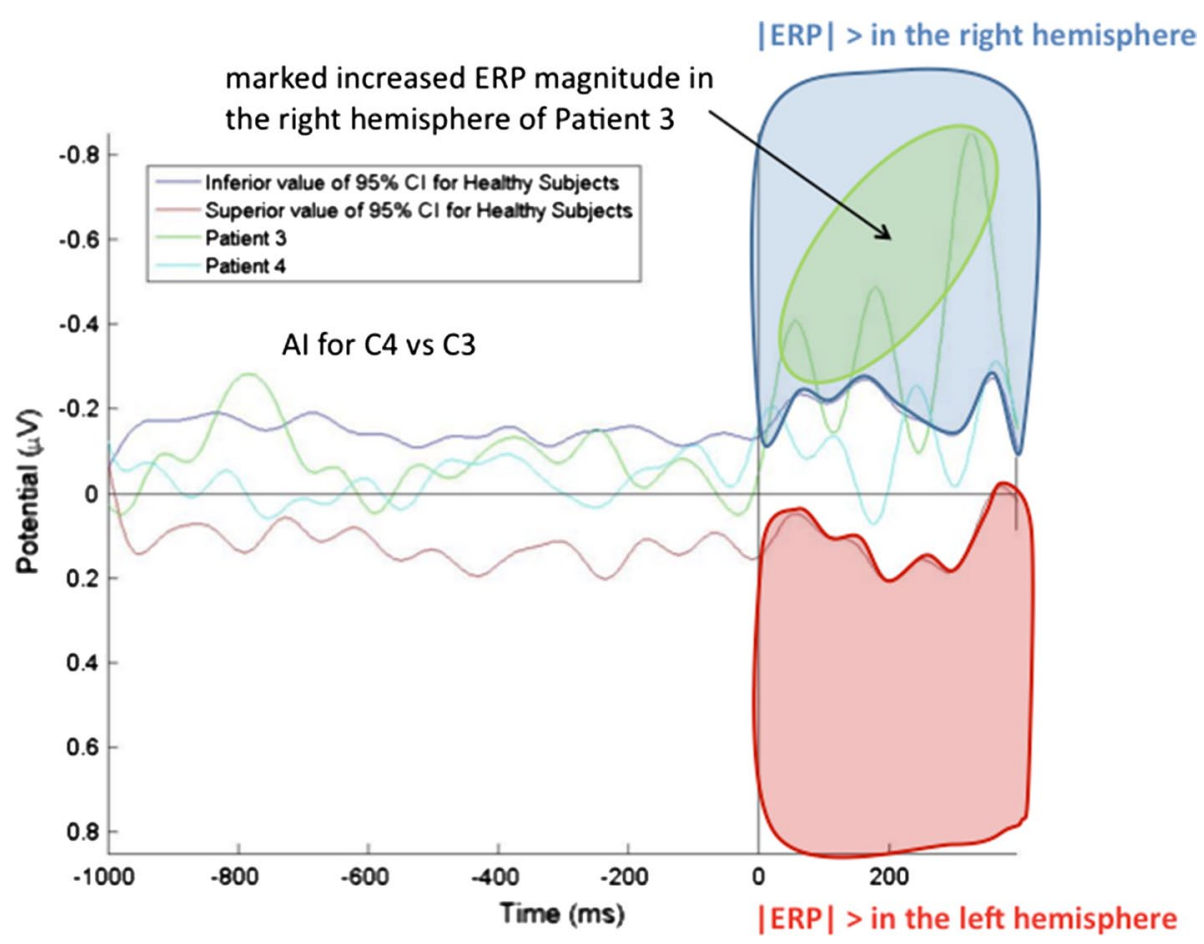

Fig. 2 Illustration of the AIs computed for two patients (P4: cyan line; P3: green line) for a single pair of homologous electrodes (e.g. C3-C4), in comparison with the upper bound (red line) and lower bound (blue line) of the $95 \%$ CI determined for the same pair of homologous electrodes in control subjects. Negative values (i.e. below the lower bound of the $95 \%$ CI) after the "go" signal $(t=0 \mathrm{~ms})$ therefore correspond to a greater ERP amplitude in the right (injured) hemisphere than in the left (healthy) hemisphere. Con-

the normalized ERP amplitude in the right hemisphere (at $\mathrm{C} 4$, for instance) was lower to that of the homologous site (C3) and that the asymmetry exceeded that observed in healthy subjects. It is important to note that the AI was then used qualitatively to determine the location and number of homologous pairs of electrodes that showed a right versus left imbalance in the ERP amplitude. The method is illustrated in Fig. 2.

\section{Results}

Behavioural data

For each experimental condition, we compared each patient's individual performances with the $95 \%$ CI from the control group. As shown in Fig. 3, the post-operative values indicated a general slowing of reaction times for patients P1 (with extensive temporal lobe resection) and P2 (with extensive frontal lobe resection). Despite having undergone extensive resection of the frontal lobe, P3 exhibited normal reaction times post-operatively (but not versely, positive values (i.e. above the upper bound of the $95 \% \mathrm{CI}$ ) in the same time window correspond to a greater ERP amplitude in the left (healthy) hemisphere than in the right hemisphere. It should be noted that (1) Note that the $y$-axis' negative and positive values are inversed (as in the classical representation of ERPs) and (2) the baseline ERP signal (before $t=0 \mathrm{~ms}$ ) remained roughly the same because since AI was close to zero during this time interval. AI asymmetry index, $E R P$ event-related potential, $C I$ confidence interval

pre-operatively). For the two patients with smaller lesions, reaction times were normal for P5 and even abnormally shorter for P4. By comparing data for the right and left hands, we found that reaction times for the impaired (left) hand were abnormally slow for $\mathrm{P} 1$ in the unimanual condition $(p<.05)$, but not in the bimanual condition. Functional asymmetry between the right and left hands was much less marked for patients P2 to P5.

\section{Event-related potential data}

For each patient and each experimental condition, we first computed the overall number of electrodes with an AI outside the $95 \%$ CI for controls. These data are summarized in Fig. 4. We found that there were more electrode sites with increased right (injured) hemisphere amplitudes than with increased left hemisphere amplitudes. This was particularly true for the two patients with the smallest lesions in the $\mathrm{L}+\mathrm{RH}-L S$ and $\mathrm{L}+\mathrm{RH}-C S$ conditions (black circles in Fig. 4). In contrast, the two patients with the frontal lesions (P2 and P3) had more sites with increased left hemisphere amplitudes in the $\mathrm{RH}-R S$ and $\mathrm{RH}-L+R S$ 

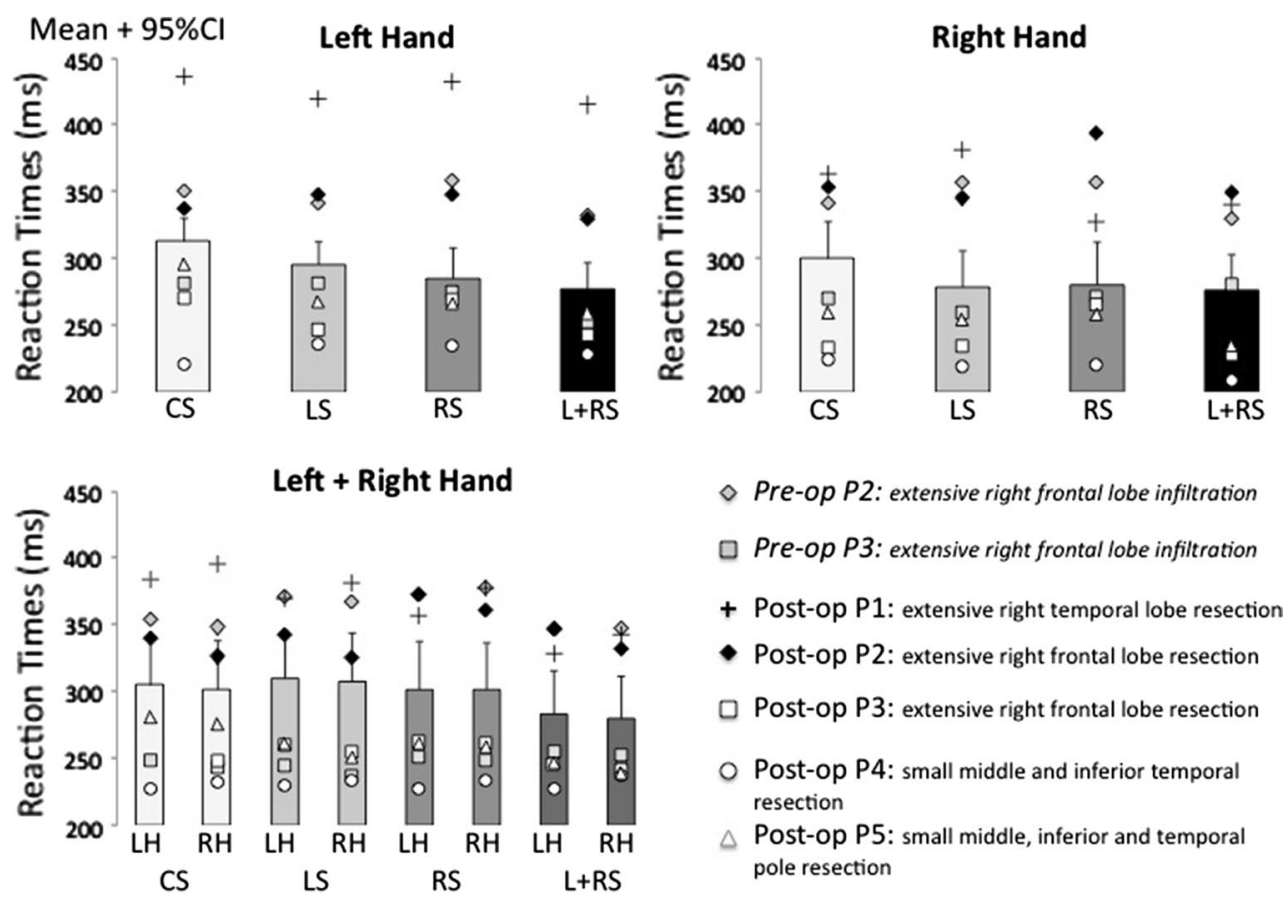

Fig. 3 Reaction times for control subjects (histograms: means $\pm 95 \%$ $\mathrm{CI})$ and the patients (geometric forms: individual means) in each of the experimental conditions. $C I$ confidence interval, $C S$ central stimulus,

$L S$ left stimulus, $R S$ right stimulus, $L+R S$ left + right stimulus; $L H$ left hand, $R H$ right hand

conditions (grey circles in Fig. 4); this was especially true for P2.

Next, we investigated the increased excitability of the injured hemisphere observed in P4 and P5. In particular, we sought to determine the topological organization of the AIs that were outside the $95 \% \mathrm{CI}$ in the two most characteristic experimental conditions $(\mathrm{L}+\mathrm{RH}-L S$ and $\mathrm{L}+\mathrm{RH}-C S$ ). As shown in Fig. 5, increased excitability in an area centred on the right motor cortex was observed in both patients.

Similarly, we sought to determine which areas of P2's healthy (left) hemisphere exhibited increased excitability. As shown in Fig. 6, we found that the main increase in cortical excitability was located in the left hemisphere. Several sites in the contralesional frontal areas demonstrated increased excitability after surgery, although similar increases were also present before surgery (Fig. 4). It must be borne in mind that this patient had already undergone brain surgery prior to the present study. One could hypothesize that this contralesional increase in cortical excitability was artifactual and was due to the difference between ERPs measured over non-resected and resected areas. However, this is unlikely because ERP amplitudes measured over the contralesional frontal areas were even greater than those measured over the dorsal areas in the same experimental condition (Fig. 7). Furthermore, the fact that the increase in cortical excitability in the contralesional frontal part was

observed (1) before surgery and (2) for a small subset of the (randomized) experimental conditions indicates that it was not an artefact in P2.

\section{Discussion}

We studied five patients after wide-awake surgery for slowgrowing tumours, in order to establish whether interhemispheric imbalance and asymmetries in cortical excitability could be measured by comparing left and right hemisphere ERP amplitudes. Furthermore, we sought to determine whether the measured changes in interhemispheric excitability would mirror (at least to some extent) estimations given by combination for the stimulus site and the hand used to react to the stimulus.

The methodology that we developed to measure changes in cortical excitability is easy to use in a clinical setting, since we simply computed interhemispheric differences in ERP magnitudes during an ecological paradigm. To the best of our knowledge, this is the first attempt to use this type of a method. This ERP-based method may be especially useful for patients having undergone wide-awake surgery, in whom interhemispheric dynamics are considerably modified.

Our results demonstrated that increased ERP magnitudes could be observed in the central part of the right 


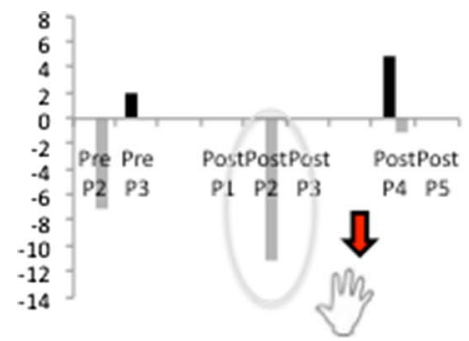

RH-RS

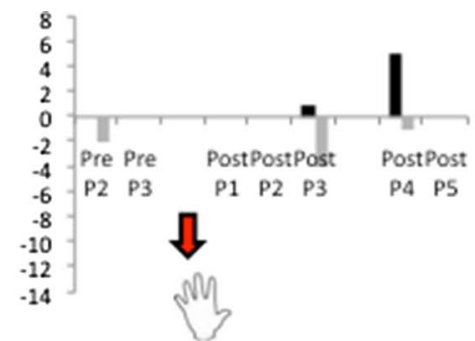

LH-LS

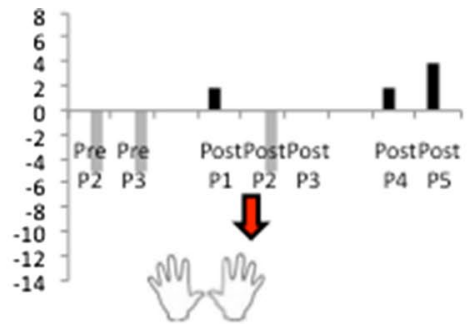

L+RH-RS

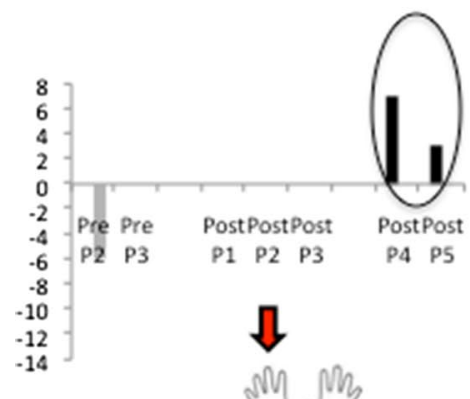

L+RH-LS

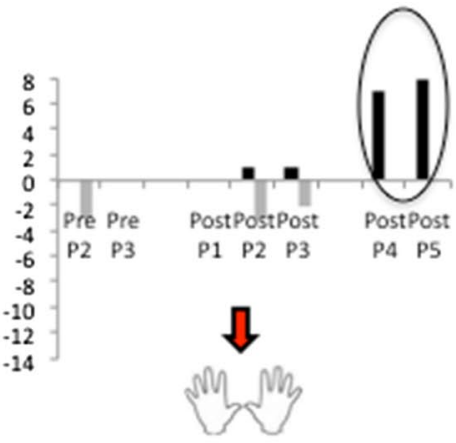

L+RH-CS

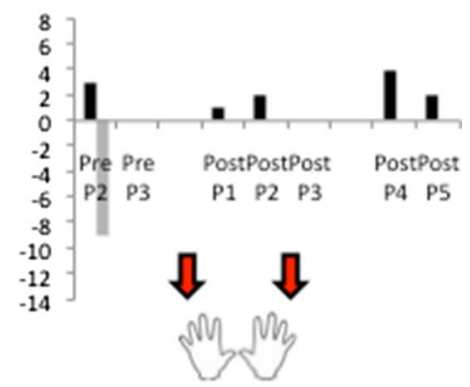

$\mathbf{L}+\mathbf{R H}-\mathbf{L}+R S$

Number of indexes of asymmetry outside $95 \% \mathrm{Cl}$ in the right (positive, $\square$ ) and left (negative, - ) hemisphere.

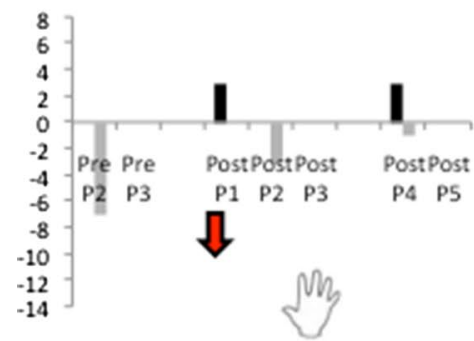

RH-LS

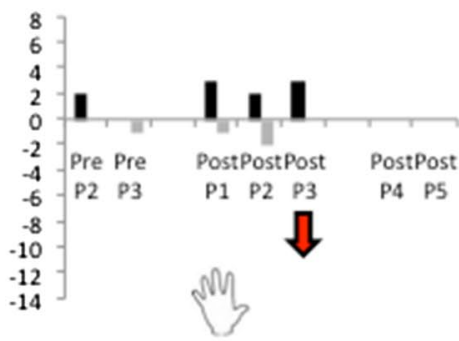

LH-RS

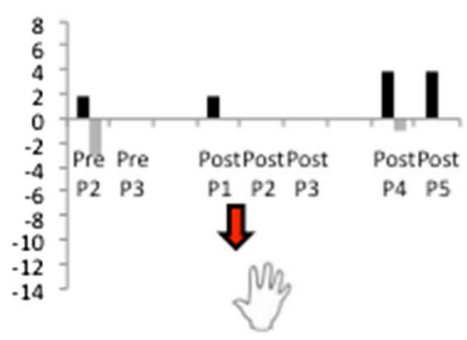

RH-CS

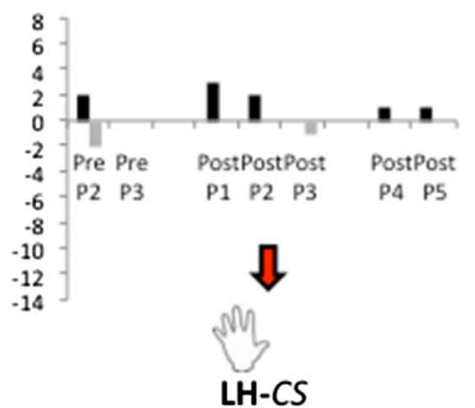

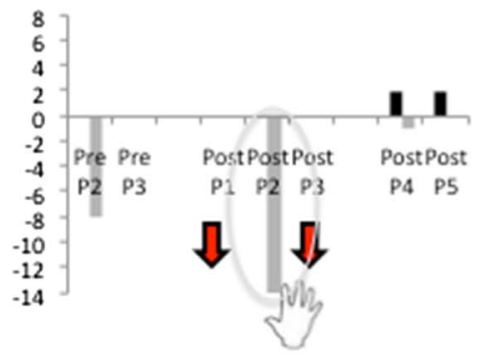

RH-L+RS

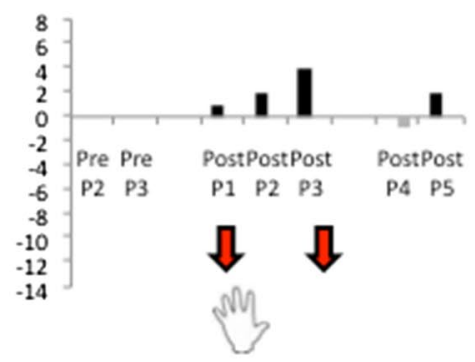

LH-L+RS

Number of indexes of asymmetry outside $95 \% \mathrm{Cl}$ in the right (positive, $\square$ ) and left (negative,, ) hemisphere.

Fig. 4 Number of electrode sites with an AI outside the $95 \%$ CI in the right hemisphere (positive AI, black) and left hemisphere (negative AI, grey) for each patient and under all experimental conditions.
$C I$ confidence interval, $C S$ central stimulus, $L S$ left stimulus, $R S$ right stimulus, $L+R S$ left + right stimulus, $L H$ left hand, $R H$ right hand, $L+R H$ left + right hand 

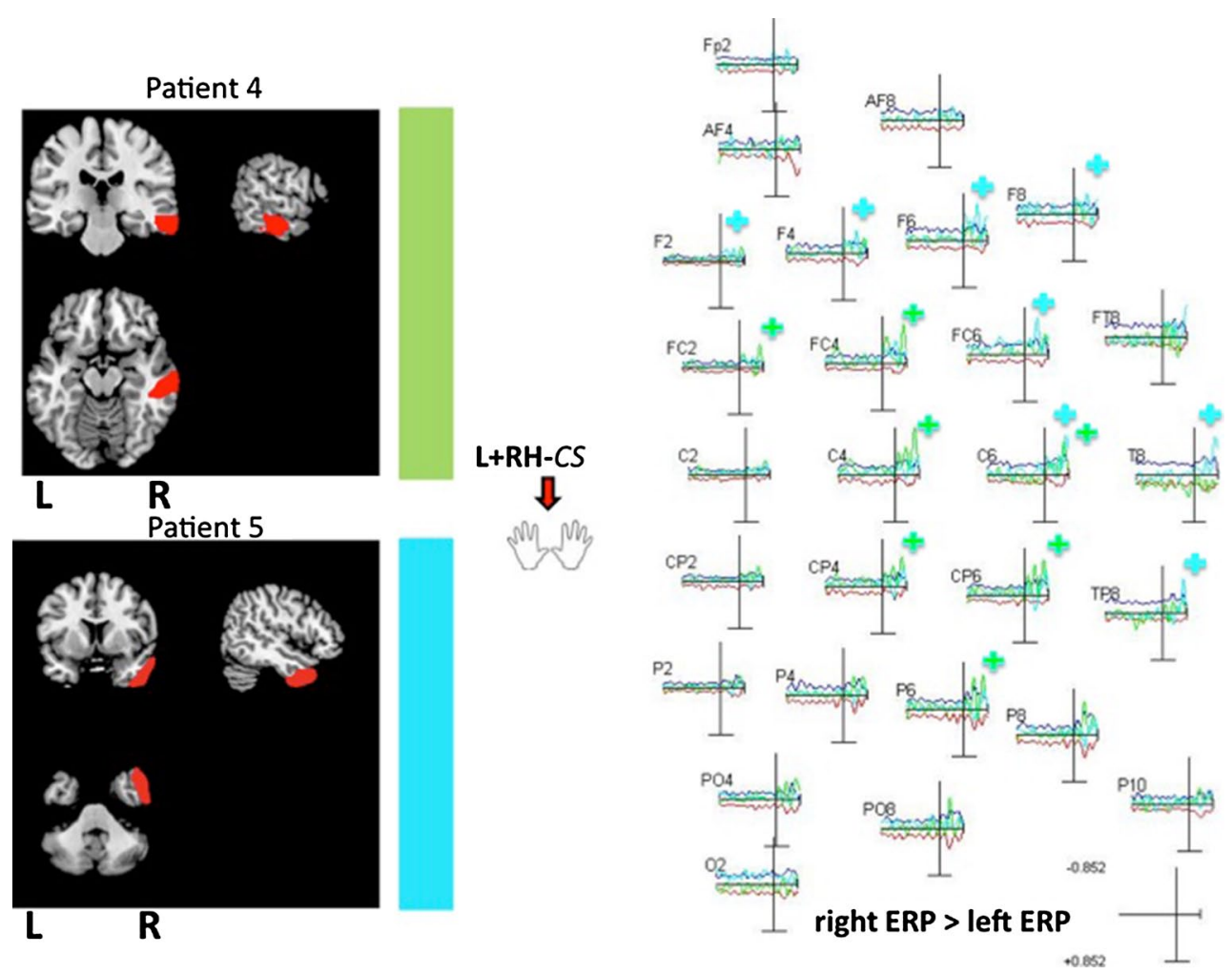

Fig. 5 Asymmetry indices computed for the two patients with the smallest lesions (P4: cyan line; P5: green line) and for each all electrode site, in comparison with the upper bound (red line) and lower bound (blue line) of the $95 \% \mathrm{CI}$ in the $\mathrm{L}+\mathrm{RH}-\mathrm{CS}$ condition. For each patient, sites for which the AI was below the lower bound of the $95 \%$ CI are indicated by the symbol "+". Increased cortical excitability was observed at several sites in the right (injured) hemisphere in these two patients with smaller lesions. For the sake of clarity, AIs are shown in the right hemisphere only. However, note that AIs represent a direct comparison of homologue pairs of electrodes between

(injured) hemisphere for the two patients with the smallest lesions. Few signs of increased excitability were observed in the healthy hemisphere for these two patients. In contrast (and to a lesser extent), increased excitability was observed in the contralesional homologue for the patients with much more extensive lesions. P2 (with a frontal lesion) exhibited clear signs of hyperexcitability in the frontal part of the healthy hemisphere both before and after the present surgery. It is important to note that two of the three patients with large resections were the most impaired in terms of reaction times. Conversely, the two patients with the smallest lesions exhibited faster reaction times than most of the control subjects (Fig. 3). For P1 (with an important temporal resection), most of the increased excitability was observed in the right hemisphere. This patient had abnormally slow reaction times, as previously observed for patients with lesions in the parietal lobe or the temporoparietal junction (Sallard et al. 2012b). In agreement with the latter study, we observed functional asymmetry (i.e.

the right and left hemisphere. A negative value (above the blue line, since the scales are inverted) meant that the normalized ERP amplitude in the right (injured) hemisphere was higher than that at the homologous site and that the asymmetry exceeded that observed in healthy subjects. Conversely, a positive value (below the red line) meant that the normalized ERP amplitude in the right hemisphere was lower to that of the homologous site and that the asymmetry exceeded that observed in healthy subjects. ERP event-related potential, $L+R H-C S$ left + right hand with the central stimulus (arrow)

longer reaction times for the left (impaired) hand than the right (lesioned) hand) in the single-handed conditions only. The opposite functional asymmetry was observed in bimanual conditions (i.e. longer reaction times for the right (healthy) hand (Fig. 3). On the whole, behavioural performances were independent of lesion size but hand responses appeared to be more affected when the parietal lobe or the temporoparietal junction had been extensively resected. This observation suggests that the deeper the surgery in this part of the brain, the more the white matter pathways required for hand rapid responses to visual stimuli are disconnected. In other words, when the resection mainly involves "U" fibres (i.e. local connectivity) at the surface of the parietal lobe or in the temporoparietal junction, behavioural performances are not impaired - as demonstrated for the two patients with the smallest lesions. Our results show that increases in ERP magnitudes and cortical excitability can be observed in the injured hemisphere after wide-awake surgery when the volume of tumour resection is low (7.1 or 


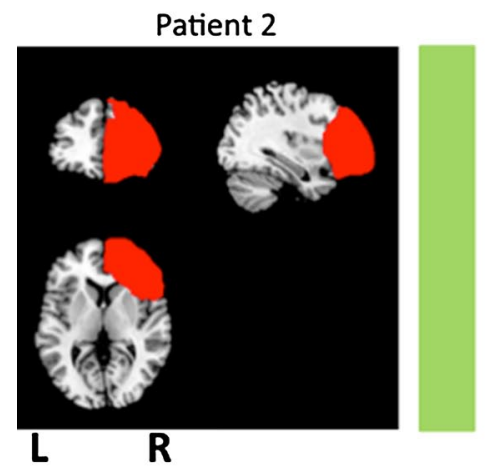

RH-RS

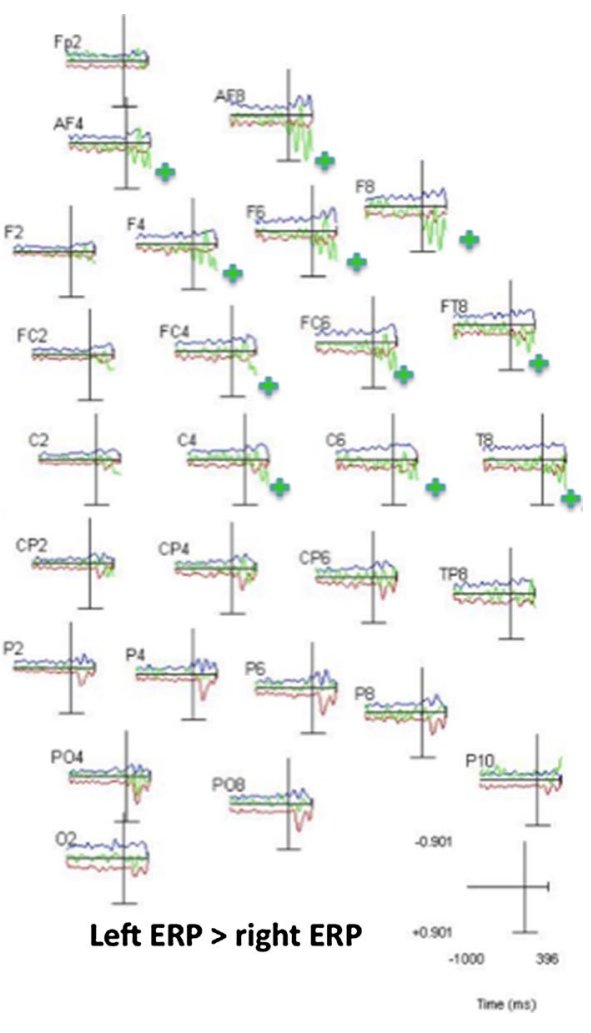

hemisphere. A negative value (above the blue line, since the scales are inverted) meant that the normalized ERP amplitude in the right (injured) hemisphere was higher than that at the homologous site and that the asymmetry exceeded that observed in healthy subjects. Conversely, a positive value (below the red line) meant that the normalized ERP amplitude in the right hemisphere was lower to that of the homologous site and that the asymmetry exceeded that observed in healthy subjects. $E R P$ event-related potential, $R H-R S$ right hand with the left stimulus (arrow)

excitability in the injured hemisphere may be limited by the lesion's extent and boundaries. We were surprised to observe that for patients with small lesions, there were more ERP increases in the right (injured) hemisphere in the condition that (in theory) would have minimized interhemispheric imbalance $(\mathrm{L}+\mathrm{RH}-C S)$. Indeed, since both hands were involved, the reciprocal interhemispheric inhibitions would supposedly be symmetrical. Given that the stimulus was presented in the sagittal axis, symmetrical visual processing would have also been expected. At the EEG level, bimanual coordination seems particularly challenging for interhemispheric balance in these patients. More exactly, simultaneously coordination of the two hands would maximize interhemispheric transfer. In such a case, the motor network in the injured hemisphere may saturate in response to increased input and because of the reorganization in functional connectivity. This saturation may induce an increase in ERP amplitude and cortical excitability. In summary, one can consider that increased ERP amplitude reflects difficulties in separating and local disinhibition may be prevented. Increases in cortical 


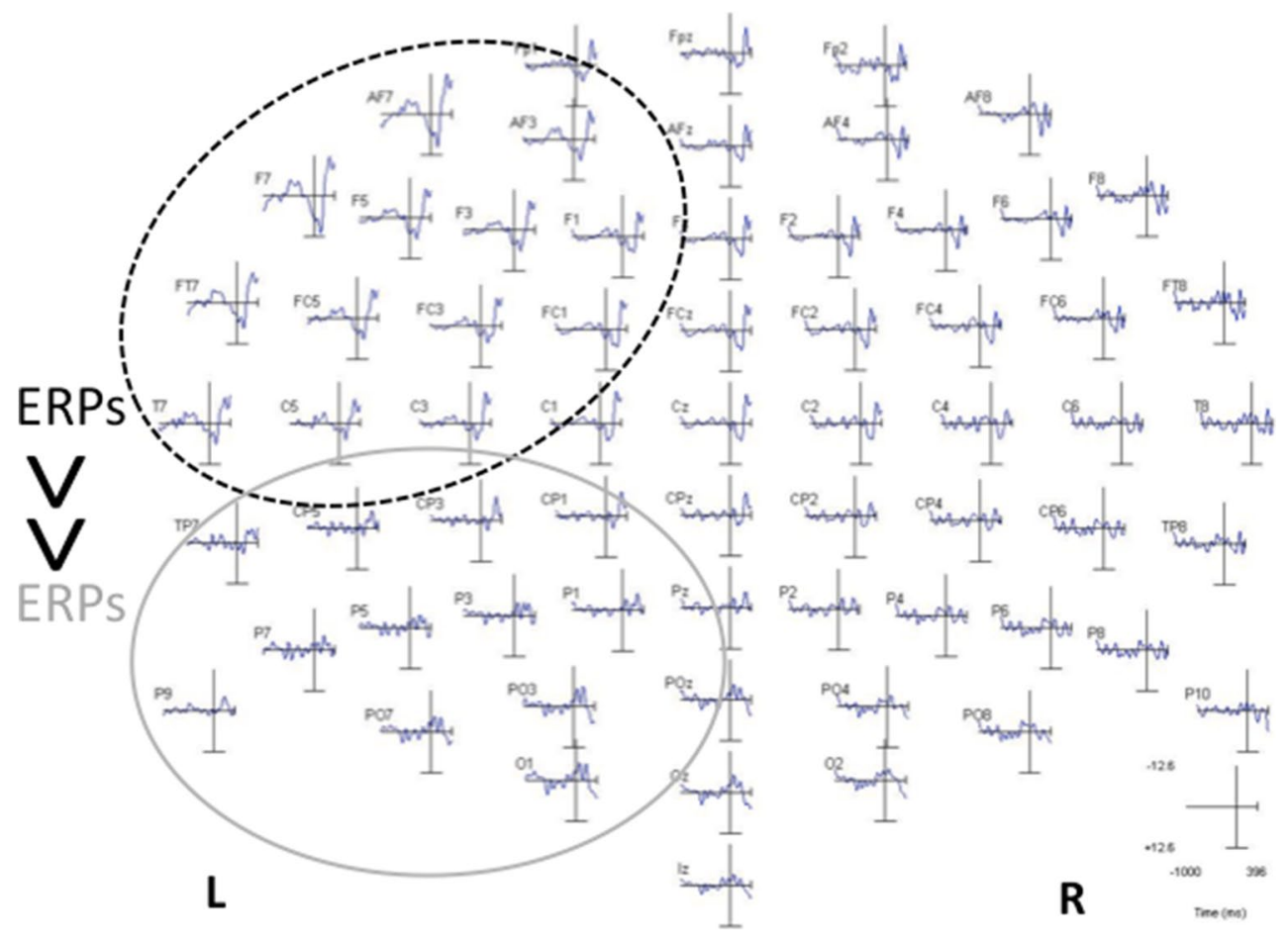

Fig. 7 Data on all ERPs for P2 in the RH - RS condition, showing that ERP amplitudes were already greater in frontal sites than in dorsal sites before the AIs were computed. The value of this control experiment was further strengthened by the fact that increases in ERP amplitude were never observed in the contralesional frontal homologue under any of the experimental conditions. We therefore concluded that the observed increases in frontal sites were not artifactual. $E R P$ event-related potential, $R H-R S$ right hand with the right stimulus (arrow) processing the sources of information that arise in each of the two hemispheres and converge towards a smaller network. More specifically, local inhibition may become less effective. This hypothesis could be tested by using pairedpulse TMS paradigms to assess intracortical inhibition.

We were intrigued to read that in patients with extensive lesions of the right temporoparietal junction (that overlap with lesions observed in our patients), bilateral stimulation (whether visual or tactile) induced the extinction of the visual stimuli applied to the impaired hemifield or hemibody (but not those applied to the healthy ones) (Driver and Vuilleumier 2001). Similarly, we found the bilateral and symmetrical stimulation was the most challenging paradigm in our two patients with small lesions (in the right middle and inferior temporal area and in the temporal pole, respectively) - despite the fact that these patients did not exhibit any sign of visual extinction. As shown previously (Sallard et al. 2012b) and in the present study, major lesions in the temporoparietal junction induce a general increase in reaction times for both hands; however, the increase is greatest for the healthy (right) hand when both hands are used synchronously in bilateral conditions. The opposite results are observed under unilateral conditions. Hence, one can hypothesize that extensive lesions to the right temporoparietal junction or to a distributed network involving the arcuate fasciculus and its ramifications in the temporal lobe result in desynchronization of both the right and left hands. More exactly, it suggests that this right distributed temporal network (especially the deeper part) set the pace for the left hemisphere and synchronizes the right hand with the left hand during simultaneous bimanual movements. Right hand movement may depend on that of the left hand. We did not observe these effects in patients with more frontal lesions (i.e. the left hand was more impaired in all conditions). It is also intriguing to note that for patients with smaller lesions in the right middle and inferior temporal area and in the temporal pole, respectively, hand movements on both body sides were fast and well synchronized-even though excitability was greater in the right hemisphere only. The latter increase may have been required to maintain bimanual synchronization.

We observed a marked increase in excitability in the contralesional homologue for a patient with an extensive frontal lesion (P2). Importantly, these increases did not appear to be artifactual and may evidence compensation by the contralesional frontal hemisphere. However, the reasons for this increase in cortical excitability are more difficult to interpret, since this phenomenon was not observed 
in the other patient with a frontal lesion. These increases in the contralesional frontal hemisphere were observed for two conditions that (in theory) were not supposed to be particularly challenging for the right (injured) hemisphere (RH $-R S$ and $\mathrm{RH}-L+R S$ ) because they should have involved the left (healthy) perceptual-motor network.

The present study had several limitations. The sample size was small because the pre- and post-operative assessments were lengthy and burdensome. Furthermore, the tumour size and site varied markedly from one patient to another. To circumvent these limitations, we used a differential approach in which individual performance levels were compared with a baseline. For each condition, each patient's performance was compared with the $95 \%$ CI derived from a control group. Differential approaches are particularly appropriate when between-subjects variability is large (as in many clinical studies) and so are fully justified in the present case. This approach was even more justified when the variability in the lesion extent and size is considered. It is important to bear in mind that lesion variability is always present in studies of patients with brain lesions. Even though researchers try to control for lesion size and locations, lesion reconstruction (when performed) is not usually accurate enough to determine which white matter pathways are injured, for instance. Furthermore, as demonstrated by functional mapping of the brain, there is also huge between-subjects variability in anatomical landmarks and functional performance. In many cases, datasets from individual patients are averaged—constituting a major limitation. Given that white matter pathways link remote brain areas with various functions, small differences in the state of damage may lead to complete different functional states. Accordingly, we consider that our differential approach was justified for determining whether it was possible to measure changes in interhemispheric electrophysiological balance after wide-awake surgery.

With our method, the ERP amplitudes in the two hemispheres are symmetrical before the stimulus, but are modified afterwards; we can therefore consider that the interhemispheric excitability has been modified. For a given stimulus intensity and baseline EEG signal, increases in excitability are defined as an increase in the amplitude of response. Classically, changes in cortical excitability in the motor domain and in sensory domains (visual and auditory domains, etc.) are studied by measuring evoked potentials. Furthermore, evoked potentials are always a combination of multiple sources and generators. EEG signals will always have several sources. Lastly, the presence of a preparatory period (due to the fixation cross prior to the appearance of the visual stimulus) does not invalid our methodology and results. For each patient and each experimental condition, we determined whether the ERP values were abnormal (i.e. outside the $95 \% \mathrm{CI}$ derived from a control group) after the stimulus onset. Conversely, we checked that the ERP values were normal (i.e. within the $95 \%$ CI) before the stimulus onset. In other words, any pre-motor potential that may have occurred was symmetrical in the two hemispheres. In contrast, we observed asymmetrical ERP values when comparing the hemispheres after the "go" signal.

The type of surgical procedure (wide awake vs. anesthetized) should not have any influence on the results. However, the wide-awake procedure allows a functional mapping of cortical and subcortical areas around the tumour using direct electrical stimulation. As such, the resection is more precise and can preserve the connectivity online much more than classical procedures with anaesthesia. Using our method, the hypothesis that identical results and changes in local excitability could also be observed, with more classical surgical procedures (and for different nature of the lesion), is appealing, but it needs to be demonstrated.

To the best of our knowledge, this is the first study to have compared interhemispheric ERP amplitudes with a view to detecting asymmetries in cortical excitability. Our simple method may have value as a clinical tool for assessing and then monitoring interhemispheric electrophysiological imbalance in these patients with a slow-growing tumour. More particularly, this measurement method may have clinical value for determining which environmental and visuo-manual task constraints induce interhemispheric electrophysiological asymmetries (especially if behavioural measurements are not enough accurate). This simple approach may help to fine-tune rehabilitation and occupational therapy by identifying the situations that perturb the patient's interhemispheric balance. Our results also allowed strengthening a number of hypotheses concerning recovery and plasticity in patients with slow-growing lesions (Bonnetblanc et al. 2006; Desmurget et al. 2007). We found that the smaller the lesion, the more the ipsilesional hemisphere was involved in the recovery. Conversely, the greater the lesion, the more the contralesional hemisphere was involved. For patients with smaller lesions, the interhemispheric imbalance in excitability is the opposite of what is observed in stroke patients (especially during the chronic phase of stroke) (Hummel and Cohen 2005, 2006). With a view to better understanding brain plasticity and neuromodulation, there is a continued need to study how slowgrowing lesions induce recovery processes and influence brain dynamics.

Acknowledgments This study was funded by the Association pour la Recherche sur le Cancer charity (Grant 3184). We thank the patients for their participation in the study. We also thank the three anonymous reviewers for their constructive and stimulating comments about the present work.

Conflict of interest None. 


\section{References}

Andersen S, Rapcsak SZ M, Beeson PM (2010) Cost function masking during normalization of brains with focal lesions: still a necessity? NeuroImage 53(1):78

Bonnetblanc F, Desmurget M, Duffau H (2006) Low grade gliomas and cerebral plasticity: fundamental and clinical implications. Med Sci (Paris) 22:389-394

Brett M, Leff AP, Rorden C, Ashburner J (2001) Spatial normalization of brain images with focal lesions using cost function masking. NeuroImage 14(2):486

Desmurget M, Bonnetblanc F, Duffau H (2007) Contrasting acute and slow-growing lesions: a new door to brain plasticity. Brain 130:898-914

Driver J, Vuilleumier P (2001) Perceptual awareness and its loss in unilateral neglect and extinction. Cognition 79:39-88

Duffau H (2005) Lessons from brain mapping in surgery for lowgrade glioma: insights into associations between tumour and brain plasticity. Lancet Neurol 4:476-486

Finger S (1978) Lesion momentum and behaviour. In: Finger S (ed) Recovery from brain damage: research and theory. Plenum Press, New York, pp 135-164

Finger S, Stein D (1982) Brain damage and recovery: research and clinical perspectives. Academic Press, Orlando

Heiss WD, Thiel A, Kessler J, Herholz K (2003) Disturbance and recovery of language function: correlates in PET activation studies. Neuroimage 20(Suppl. 1):S42-S49

Herbet G, Lafargue G, Bonnetblanc F, Moritz-Gasser S, Duffau H (2013) Is the right frontal cortex really crucial in the mentalizing network? A longitudinal study in patients with a slow-growing lesion. Cortex doi:10.1016/j.cortex.2013.08.003
Hummel FC, Cohen LG (2005) Drivers of brain plasticity. Curr Opin Neurol 18:667-674

Hummel FC, Cohen LG (2006) Non-invasive brain stimulation: a new strategy to improve neurorehabilitation after stroke? Lancet Neurol 5:708-712

Jacobs KM, Donoghue JP (1991) Reshaping the cortical motor map by unmasking latent intracortical connections. Science 251:944-947

Krainik A, Duffau H, Capelle L, Cornu P, Boch AL, Mangin JF et al (2004) Role of the healthy hemisphere in recovery after resection of the supplementary motor area. Neurology 62:1323-1332

Netz J, Ziemann U, Homberg V (1995) Hemispheric asymmetry of transcallosal inhibition in man. Exp Brain Res 104:527-533

Nudo RJ, Wise BM, SiFuentes F, Milliken GW (1996) Neural substrates for the effects of rehabilitative training on motor recovery after ischemic infarct. Science 272:1791-1794

Sallard E, Duffau H, Bonnetblanc F (2012a) Ultra-fast recovery from right neglect after 'awake surgery' for slow-growing tumor invading the left parietal area. Neurocase 18:80-90

Sallard E, Barral J, Duffau H, Bonnetblanc F (2012b) Manual reaction times and brain dynamics after 'awake surgery' of slowgrowing tumours invading the parietal area. A case report. Brain Inj 26:1750-1755

Sanes JN, Donoghue JP (2000) Plasticity and primary motor cortex. Ann Rev Neurosci 23:393-415

Stein DG, Finger S, Hart T (1983) Brain damage and recovery: problems and perspectives. Behav Neural Biol 185:222-237

Thiel A, Habedank B, Winhuisen L, Herholz K, Kessler J, Haupt WF et al (2005) Essential language function of the right hemisphere in brain tumor patients. Ann Neurol 57:128-131 